HNO 2014 · 62:241-241

DOI 10.1007/s00106-014-2854-0

Online publiziert: 8. April 2014

c) Springer-Verlag Berlin Heidelberg 2014

M. Ptok ${ }^{1,2}$

${ }^{1}$ Klinik und Poliklinik für Phoniatrie und Pädaudiologie, Medizinische Hochschule Hannover

${ }^{2}$ Schule für Logopädie, Zentrum Schulen, Medizinische Hochschule Hannover

\title{
Praxisnahe Informationen zum Spracherwerb
}

Eine gewisse Schwierigkeit kann dabei dadurch entstehen, dass das notwendige linguistische Basiswissen kaum im Medizinstudium vermittelt wird, und das in der Schule seinerzeit erworbene Wissen um die Grammatik ist vermutlich schon vergessen. Hier denke man z. B. nur an die scheinbar simplen Fragen, welche Formen der Pluralmarkierung und welche Wortarten es im Deutschen gibt. Ein solches Basiswissen ist aber erforderlich, wenn die niedergelassene Ärztin/der niedergelassene Arzt entscheiden soll, welche Defizite denn genau bei einem sprachentwicklungsgestörten Kind zu diagnostizieren und welche Therapien zu empfehlen sind. Denn letztlich wird überwiegend in der ärztlichen Praxis entschieden, ob eine Therapieverordnung im Rahmen der Hilfs- und Heilmittel auszustellen ist oder nicht. Die Beiträge Erwerb morphosyntaktischer Fähigkeiten im Hinblick auf die Konstituentenverschiebung in die Komplementiererposition und Wortschatzerwerb, wollen, quasi als Repetitorium, eine Hilfestellung geben, Aspekte des normalen Erwerbs syntaktischer bzw. lexikalischer Fähigkeiten zu verstehen.

Die medizinischen Fachrichtungen, z. B. die Kinderheilkunde, die HNO-Heilkunde und die Phoniatrie/Pädaudiologie werden in die Betrachtungen um den Spracherwerb i. d. R. dann einbezogen, wenn es um den gestörten Spracherwerb geht. Nicht nur Normabweichungen im zeitlichen Verlauf, sondern auch qualitative Deviationen vom normalen Verlauf unterschiedlicher Laut- und Satzbildung und die Verwendung grammatikalischer Konstrukte sind zu beschreiben. Der zugrunde liegende gestörte Prozess sollte identifiziert und bezüglich der Therapiebedürftigkeit bewertet werden. Nur so kann eine defizitgerichtete, altersgemäße Behandlung definiert werden.

\section{》) In der ärztlichen Praxis wird über eine Therapieverordnung bei gestörtem Spracherwerb entschieden}

Erfreulicherweise sind gerade in den letzten Jahren einerseits deutliche Wissenszuwächse um den Spracherwerb insgesamt erzielt und andererseits Testverfahren zum Einsatz bei gestörtem Spracherwerb entwickelt worden. Somit kann die für die Praxis relevante Frage, ob ein Kind altersgemäß spricht oder eine Therapie zulasten der GKV erhalten muss, heute besser als noch vor einigen Jahren beurteilt werden
- auch wenn fast jeder „intuitiv“ zu ahnen glaubt, ob ein Kind altersgemäß sprachliche Kompetenzen erworben hat. Ganz praxisnah wird in der Arbeit Leitliniengerechte Diagnostik bei Spracherwerbsstörungen dargestellt, wie die Sprachstandserhebung konform zu der aktuellen Leitlinie, an deren Erstellung u. a. die Deutsche Gesellschaft für HNO-Heilkunde und die Deutsche Gesellschaft für Phoniatrie und Pädaudiologie mitgewirkt haben, durchgeführt werden kann.

Ziel dieses Schwerpunkthefts ist es somit, einige Themen der Spracherwerbsforschung aus klinischer Sicht anzureißen. Hierbei sollen auch einige Grundlagen repetiert und Implikationen für die klinische Praxis geschildert werden. Die Autorinnen und Autoren würden sich freuen, wenn das Interesse am faszinierenden Phänomen Erstspracherwerb, soweit nicht schon vorhanden, mit den Beiträgen geweckt worden ist.

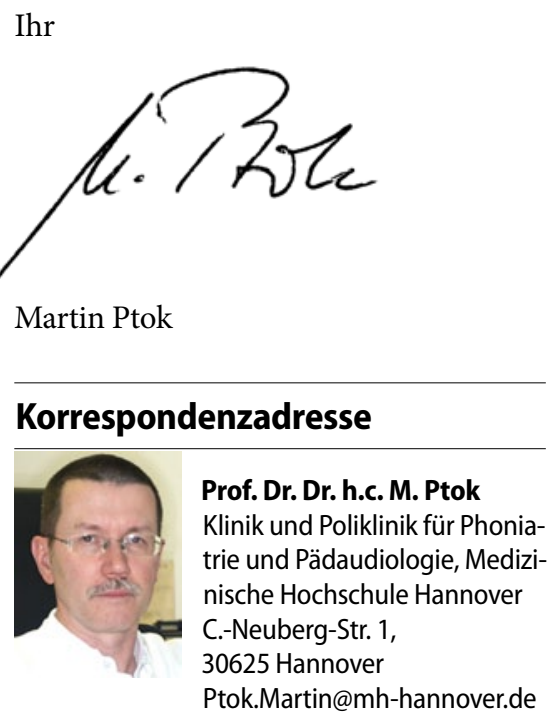

Interesssenkonflikt. M. Ptok gibt an, dass kein Interessenkonflikt besteht. 